\title{
Why radiography should no longer be considered a surrogate outcome measure for longitudinal assessment of cartilage in knee osteoarthritis
}

\author{
Ali Guermazi*1, Frank W Roemer', Deborah Burstein² and Daichi Hayashi'
}

\begin{abstract}
Imaging of cartilage has traditionally been achieved indirectly with conventional radiography. Loss of joint space width, or 'joint space narrowing', is considered a surrogate marker for cartilage thinning. However, radiography is severely limited by its inability to visualize cartilage, the difficulty of ascertaining the optimum and reproducible positioning of the joint in serial assessments, and the difficulty of grading joint space narrowing visually. With the availability of advanced magnetic resonance imaging (MRI) scanners, new pulse sequences, and imaging techniques, direct visualization of cartilage has become possible. MRI enables visualization not only of cartilage but also of other important features of osteoarthritis simultaneously. 'Pre-radiographic' cartilage changes depicted by $\mathrm{MRI}$ can be measured reliably by a semiquantitative or quantitative approach. MRI enables accurate measurement of longitudinal changes in quantitative cartilage morphology in knee osteoarthritis. Moreover, compositional MRI allows imaging of 'pre-morphologic' changes (that is, visualization of subtle intrasubstance matrix changes before any obvious morphologic alterations occur). Detection of joint space narrowing on radiography seems outdated now that it is possible to directly visualize morphologic and pre-morphologic changes of cartilage by using conventional as well as complex MRI techniques.
\end{abstract}

\section{Introduction}

Hyaline cartilage is an important intra-articular structure that is prone to damage by trauma or degeneration.

\footnotetext{
*Correspondence: quermazi@bu.edu

'Quantitative Imaging Center, Department of Radiology, Boston University School of Medicine, 820 Harrison Avenue, FGH Building, 3rd Floor, Boston, MA 02118, USA Full list of author information is available at the end of the article
}

Damaged cartilage is one of the key tissues in the disease process that is understood as osteoarthritis (OA), a multi-tissue disease leading eventually to joint failure [1]. For decades, direct imaging of cartilage was not possible and investigators relied instead on indirect methods using conventional radiography. Loss of joint space width (JSW), or joint space narrowing (JSN), is considered a surrogate marker for cartilage damage [2]. In contrast, magnetic resonance imaging (MRI) allows direct visualization of cartilage, and technologic advances have led to sophisticated tools such as high-resolution morphologic imaging and compositional or biochemical assessment [3].

In this review, we will first describe the current role of conventional radiography for cartilage assessment in OA and then discuss the current and future roles of MRI for the evaluation of cartilage pathology in OA. We will explain the strengths and limitations of both imaging modalities on the basis of available evidence from the literature and elucidate why today MRI should be considered the imaging modality of choice for cartilage assessment in the context of OA of the knee joint.

\section{Imaging of cartilage by radiography}

Radiographs are used in clinical practice to establish the diagnosis of $\mathrm{OA}$ and to monitor the progression of the disease. Radiography can clearly visualize bony features, such as marginal osteophytes, subchondral cysts, and sclerosis, that are characteristic features of OA, but direct visualization of cartilage is not possible. Instead, radiography allows an indirect estimate of cartilage thickness and meniscal integrity by assessment of JSW in the patellofemoral and tibiofemoral joints.

At present, radiographic diagnosis of knee OA is based on the Kellgren-Lawrence (KL) grading system, a semiquantitative composite score that is based on two radiographic features: the presence of osteophytes and JSN [4]. Radiographic knee OA is defined as KL grade 2 or above: that is, the presence of a definite marginal osteophyte (KL grade 2), the presence of JSN (KL grade 3), or boneon-bone contact (KL grade 4) [5]. Once a knee exhibits 
JSN in either the medial or lateral tibiofemoral compartment, the knee is classified as osteoarthritic despite the absence of obvious osteophytes. Assessment of knee OA severity relies mainly on JSN and subchondral bone lesions. Increased JSN is the most commonly used criterion for defining longitudinal OA progression, and the complete loss of JSW, characterized by bone-on-bone contact on the weight-bearing anteroposterior (AP) radiograph, is one of the factors in the decision for joint replacement [2]. In contrast to the KL scheme, the Osteoarthritis Research Society International (OARSI) atlas classification scores tibiofemoral JSN and osteophytes separately in each compartment of the knee [6] by using a 4-point scale (0-3).

Radiography is available virtually everywhere and its cost is much lower than that of MRI. Imaging time is short, and there is little discomfort for the patient. Automated software that delineates the femoral and tibial margins of the joint and that calculates JSW at fixed locations has been developed, and the relative responsiveness of JSW measurements from digital knee radiographic images has been shown to be comparable to cartilage morphometry measures derived from MRI in detecting OA progression [7]. At present, radiographically detected JSN is the only structural endpoint accepted by the regulatory bodies in the US (US Food and Drug Administration, or FDA) and Europe (European Medicines Agency) to prove efficacy of disease-modifying osteoarthritis drugs (DMOADs) targeting OA in phase III clinical trials [5]. According to the recently published summary and recommendations of the OARSI FDA OA Assessment of Structural Change Working Group, radiographic JSW is still a recommended option for trials of structure modification although the construct represents a number of pathologies and trial duration may be long [8].

\section{Limitations of radiography}

The lack of reproducibility of radiographic joint space measurements in longitudinal assessment is one of the main shortcomings of radiography, especially for the extended-knee radiograph (that is, a bilateral weightbearing AP view of both knees in full extension) [5]. Changes in knee pain between examinations can introduce systematic measurement error for radiographs in full extension [2]. Mazzuca and colleagues [9] detected significant increases in tibiofemoral JSW in extendedknee radiographs taken 7 to 14 days apart of OA subjects who had experienced relief of an induced flare of knee OA pain. Notable differences in JSW may be demonstrated depending on the angulation of the knee (Figure 1). Thus, the lack of reproducibility of positioning in longitudinal studies and large multicenter trials remains a major drawback to the use of radiography.
To overcome this problem, various protocols have been proposed to enable standardized radiographic assessment of the knee [2]. Of those, the fixed flexion view with the use of a positioning frame (for example, SynaFlexer ${ }^{\mathrm{ma}}$; CCBR-SYNARC, Newark, CA, USA) [10] is the most widely used protocol in ongoing large-scale multicenter OA studies, such as the Osteoarthritis Initiative (OAI) and the Multicenter Osteoarthritis Study (MOST). Other protocols use fluoroscopy to confirm satisfactory anatomical positioning of the medial tibial plateau prior to acquisition of the radiograph $[11,12]$.

Such measures may alleviate the problems associated with positioning, but even if the image acquisition method is optimized, interpreting JSN may still be problematic. Based on data from the OAI, a recent study by Guermazi and colleagues [13] showed marked differences in thresholds for scoring of JSN between expert readers. In light of these findings, research studies using radiographic semiquantitative grading of OA should rely on centralized adjudicated reading for all grading in order to minimize variability.

The inability of radiography to directly visualize cartilage and many other OA features is the insurmountable shortcoming of this imaging modality (Figure 2). Recently, Amin and colleagues [14] examined the relationship between progression of JSN on radiographic images and cartilage loss on MRI. While their results provided longitudinal evidence that radiographic progression of JSN is correlated with cartilage loss assessed on MRI, the authors concluded that if radiography were used alone, a substantial proportion of knees with cartilage loss would be missed [14]. In longitudinal studies, radiographic progression of JSN is neither a sensitive [14] (Figures 3 and 4) nor a specific [1] (Figure 5) measure of OA disease progression when compared with MRI findings.

It was previously believed that JSN and its changes reflect only articular cartilage thinning, but several studies have shown that alterations in the meniscus, such as meniscal extrusion or subluxation, also contribute to JSN [1,15,16]. A more recent study, by Hunter and colleagues [1], compared MRI and weight-bearing posteroanterior radiographs to explore the relative contribution of several morphologic features, including cartilage, meniscal damage, and position, to the radiographically detected JSN. The authors found that a substantial proportion of the explained variance in JSN was due to meniscal position and degeneration, and a substantial proportion of change in JSN resulted from change in meniscal position.

Lastly, one should note that patients usually have medial knee OA and the regular use of JSW measurements is performed in the medial compartment. However, the lateral joint space may become widened as 


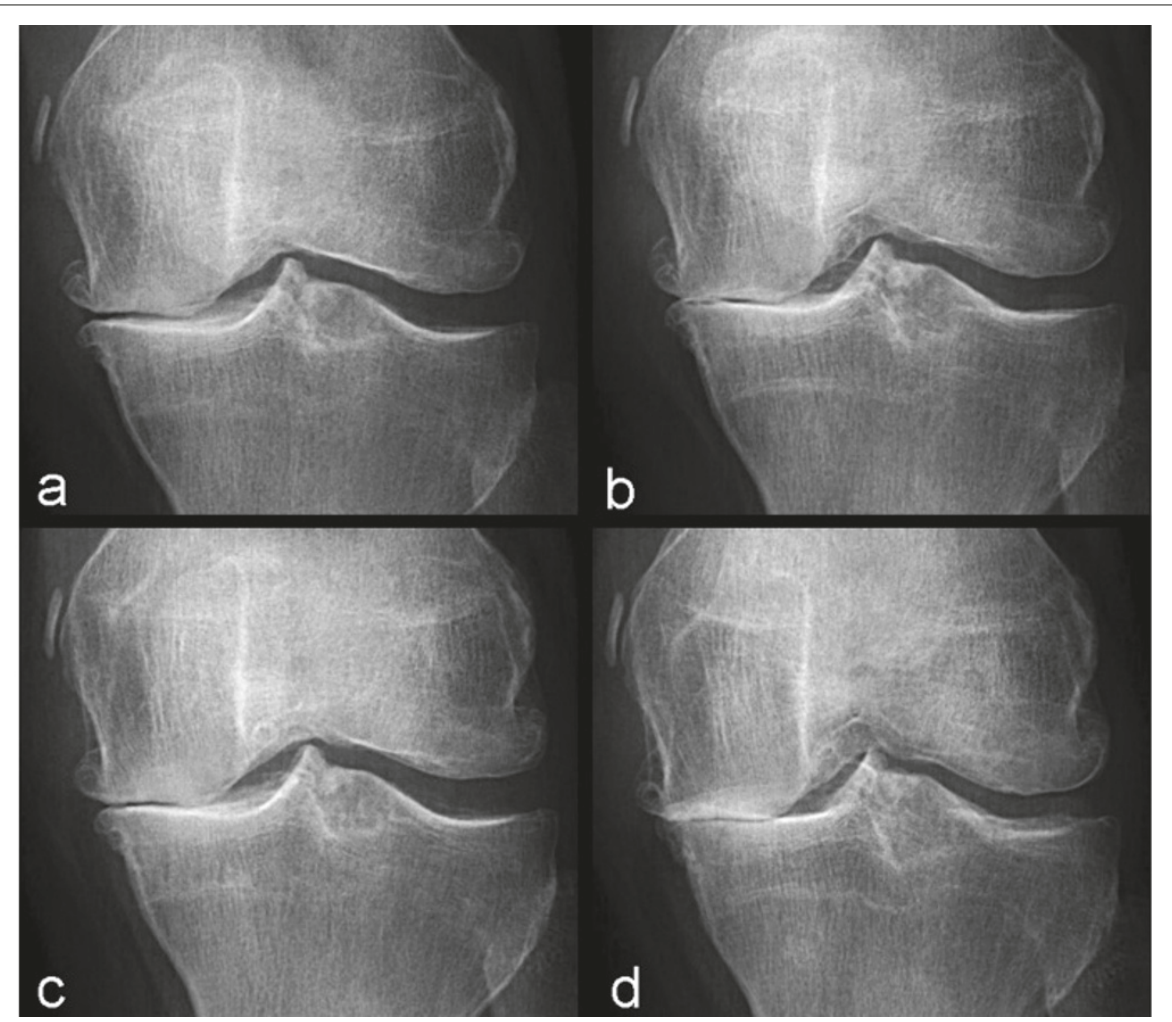

Figure 1. Radiographs at baseline and 2-year follow-up of a 61-year-old woman with osteoarthritis. At two time points, radiographs were taken with $5^{\circ}, 10^{\circ}$, and $15^{\circ}$ angulation of the knee. (a) Anteroposterior (AP) radiograph taken at $5^{\circ}$ angulation shows medial joint space narrowing (OARSI grade 2 and Kellgren-Lawrence grade 3). (b) However, AP radiograph taken at $10^{\circ}$ angulation shows OARSI grade 3 joint space narrowing (Kellgren-Lawrence grade 4). (c) Similarly, at follow-up, AP radiograph taken at $5^{\circ}$ angulation shows OARSI grade 2 joint space narrowing (KellgrenLawrence grade 3 ) whereas that taken at $15^{\circ}$ angulation (d) shows OARSI grade 3 joint space narrowing (Kellgren-Lawrence grade 4 ). If images (b) and (c) are compared longitudinally, one observes a paradoxical 'joint space widening'. This example highlights the importance of technique to ascertain the reproducibility of the same angulation of the knee in longitudinal studies. OARSI, Osteoarthritis Research Society International.

a result of severe medial JSN. In such secondary widening of joint space, articular cartilage in the lateral compartment may be normal or abnormal, but even if there is cartilage thinning, it is very difficult to assess by radiography alone [17].

\section{Imaging of cartilage by magnetic resonance imaging}

An important advantage of MRI over radiography is the ability of the former to visualize cartilage and other joint tissues directly. MRI is a powerful non-invasive tool for detecting cartilage damage and for monitoring the effects of conservative and surgical therapy [3]. Other characteristic OA features, such as bone marrow lesions, meniscal lesions, and synovitis, can be visualized and assessed simultaneously (Figure 2), enabling study of the potential OA risk factors and mechanisms of the OA disease process $[18,19]$. MRI techniques used for the assessment of cartilage can be divided broadly into two categories: morphologic and compositional evaluation [3].

\section{Morphologic assessment}

MRI techniques for morphologic assessment of knee articular cartilage provide accurate information about focal or diffuse partial- or full-thickness cartilage loss [3]. In clinical research, especially in knee OA studies, morphologic assessment of cartilage with MRI is performed by using semiquantitative scoring methods, such as the Whole Organ Magnetic Resonance Imaging Score (WORMS) [20], the Boston Leeds Osteoarthritis Knee Score (BLOKS) [21], and the Knee Osteoarthritis Scoring System (KOSS) [22]. For example, in WORMS, cartilage signal and morphology are scored on a point scale of 0 to 6 (that is, $0,1,2,2.5,3,4,5$, and 6 , where 0 is normal and 6 is diffuse full-thickness loss) in 14 articular surface regions of the knee joint. The other methods employ similar semiquantitative approaches, and details can be found in the original publications. Excellent reliability data have been published for all three scoring systems [23]. Additionally, a new scoring system (called Magnetic Resonance Imaging Osteoarthritis Knee Score, or MOAKS) has been developed to maximize the advantages 


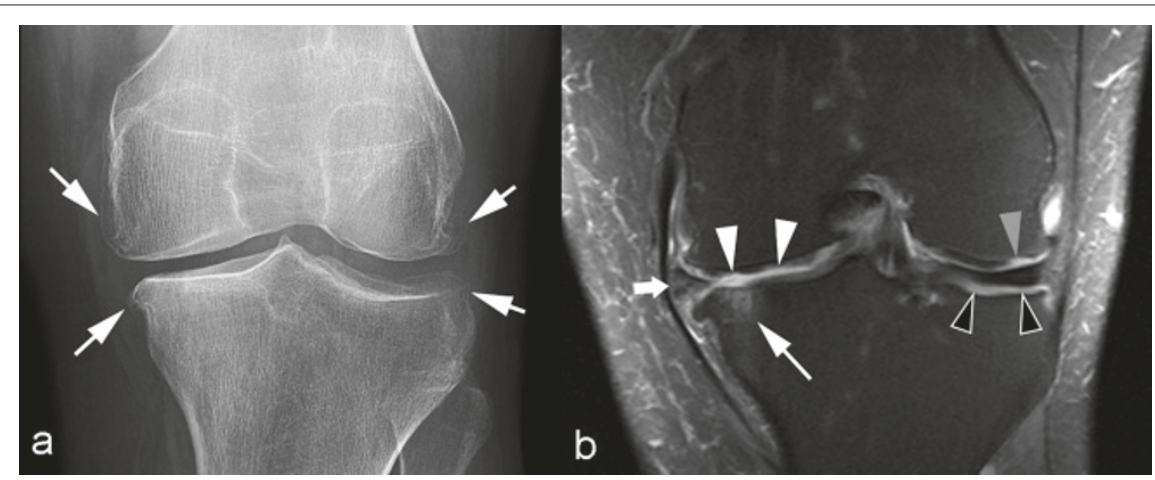

Figure 2. Comparison of an anteroposterior radiograph and a coronal magnetic resonance image of the knee. (a) Anteroposterior radiograph of the left knee demonstrates marginal osteophytes of the medial and lateral femur and tibia (arrows). Joint space width appears normal in the lateral tibiofemoral compartment, but there is mild to moderate medial tibiofemoral joint space narrowing. No other obvious bony abnormalities are seen. (b) Coronal fat-suppressed proton density-weighted magnetic resonance imaging performed on the same day reveals a subchondral bone marrow lesion (thin white arrow) at the medial tibial plateau subjacent to a focal full-thickness cartilage defect. Multiple partialthickness defects of the medial femoral condyle cartilage (white arrowheads) are also noted. Notably, focal full-thickness cartilage defects (gray arrowhead) are more extensive at the lateral femoral condyle and subchondral bone (black arrowheads) is almost completely denuded at the lateral tibial condyle, despite radiographically normal appearance of the lateral tibiofemoral joint space width. Most of the joint space narrowing of the medial tibiofemoral joint is secondary to a partially macerated and extruded medial meniscus (thick white arrow). Additionally, there is attrition of the medial and lateral tibial plateaus and marginal osteophytosis. This example demonstrates why radiography should no longer be considered a surrogate outcome measure for longitudinal assessment of cartilage in knee osteoarthritis.

and reduce the disadvantages of existing scoring systems [24].

These semiquantitative methods evaluate the morphologic characteristics of articular cartilage in conjunction with those of other intra- and periarticular structures (for example, subchondral bone, menisci, osteophytes, and synovium) in order to establish risk factors for symptoms and structural progression of disease. These features are also used as outcome measures, and cartilage is the most commonly applied [23]. Using the WORMS method, Roemer and colleagues [25] showed that, in participants with no or only minimal baseline cartilage damage, a high body mass index, meniscal damage, synovitis, or effusion or the presence of any severe baseline MRI-depicted lesions is strongly associated with an increased risk of rapid cartilage loss.

Crema and colleagues [3] recently summarized details of currently available MRI techniques for morphologic assessment (that is, conventional spin-echo (SE) sequences and gradient-recalled echo (GRE) sequences, fast spin echo (FSE) sequences, and more advanced threedimensional (3D) SE and GRE sequences). Each technique has its strengths and limitations, and the physician or researcher must consider them carefully when selecting appropriate techniques for a particular study.

Quantitative analysis of cartilage morphology exploits the 3D nature of MRI data sets to assess tissue dimensions, such as volume and thickness, or signal as continuous variables. A recent meta-analysis demonstrated that MRI can measure change in quantitative cartilage morphometry reliably and with good responsiveness [26]. Multiple MRI-based quantitative cartilage measures (for example, volume, surface area, and percentage of total area of bone not covered by cartilage) can be used, but most of these are strongly related, and it appears that some may be redundant or contain minimal additional information. Buck and colleagues [27] performed a study to identify an efficient subset of core measures which comprises a comprehensive description of cartilage morphology and its longitudinal changes in healthy and diseased cartilage. The study showed that three measures (that is, mean cartilage thickness over the total area of the subchondral bone, total subchondral bone area, and percentage of denuded area of the subchondral bone) explain over $90 \%$ of the cross-sectional and longitudinal variation in a larger set of common cartilage morphology measures in knees with or without OA. Focusing on this efficient subset of knee cartilage morphology measures should be encouraged in future studies.

Another strategy for a more efficient measurement of cartilage thickness has been proposed by Buck and colleagues [28]. The sensitivity to change of quantitative analysis of cartilage in knee OA by using MRI is compromised by the spatial heterogeneity of cartilage loss. The authors hypothesized that determining the magnitude of thickness change independently of anatomic location provided improved discrimination between healthy subjects and OA participants longitudinally. The study showed that this 'ordered values approach' is sensitive to cartilage thinning in KL grade 3 and cartilage thickening in KL grade 2 versus $K L$ grade 0 participants. 


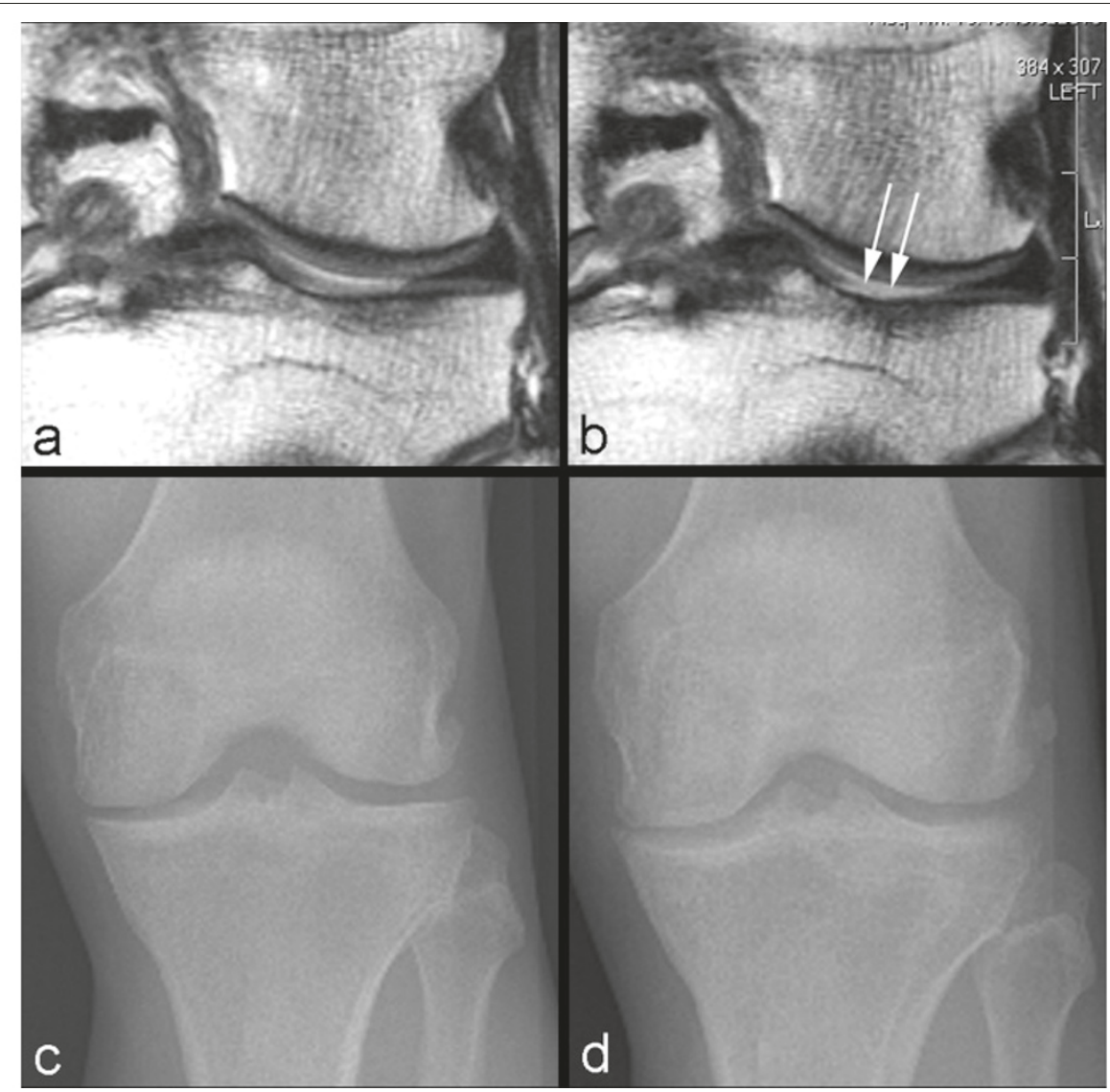

Figure 3. Example of non-sensitivity of radiography. (a) Baseline coronal intermediate-weighted magnetic resonance imaging shows hyperintensity in the weight-bearing portion of the lateral tibial plateau but no definite cartilage defect. (b) At 24-month follow-up, an incident focal full-thickness defect has developed in the corresponding area of the lateral tibial plateau (arrows). (c) Baseline anteroposterior radiograph does not show any joint space narrowing in the lateral tibiofemoral joint. (d) No change to baseline is observed at 24-month follow-up.

More recently, Wirth and colleagues [29] demonstrated that an 'extended ordered values approach' shows higher sensitivity to differences in longitudinal rates of cartilage loss in knees with and without baseline JSN than anatomical subregions and radiography. Because they circumvent the challenges of a priori selection of particular regions in clinical trials, these novel methods may become useful tools in DMOAD trials [29].

Quantitative MRI has been used in clinical trials assessing the efficacy of pharmacologic therapy of OA. In a comparison of users of cyclooxygenase- 2 inhibitors with users of conventional non-steroidal anti-inflammatory drugs, the latter group was reported to have higher knee cartilage volume loss [30]. Quantitative MRI has also been used in studies attempting to identify biomarkers for prediction of OA disease progression. Higher bone remodeling was associated with reduced cartilage loss [31], and low levels of cartilage biomarkers at baseline were associated with a significantly reduced rate of medial cartilage volume loss [32]. Higher baseline values of interleukin- $6, \mathrm{C}$-reactive protein, and cartilage oligomeric matrix protein were predictive of greater risk of cartilage volume loss [33], but over time a reduction in matrix metalloproteinase (MMP)-1 and MMP-3 levels correlated best with reduced cartilage volume loss and the effect of DMOAD treatment.

\section{Compositional assessment}

Hyaline articular cartilage is composed of a fluid-filled macromolecular network that supports mechanical loads. This macromolecular network consists mainly of collagen and proteoglycans. Collagen is the most abundant macromolecule, and aggrecan, a large aggregating proteoglycan, is the second most abundant. In normal joints, the collagen network acts as the structural framework for tissue, providing the main source of its tensile and shear strength. Glycosaminoglycans are covalently attached as side chains to the proteoglycan molecule and exhibit abundant negatively charged carboxyl and sulfate groups. Glycosaminoglycans provide the cartilage considerable compressive strength. Because proteoglycans have a substantial net negative fixed 


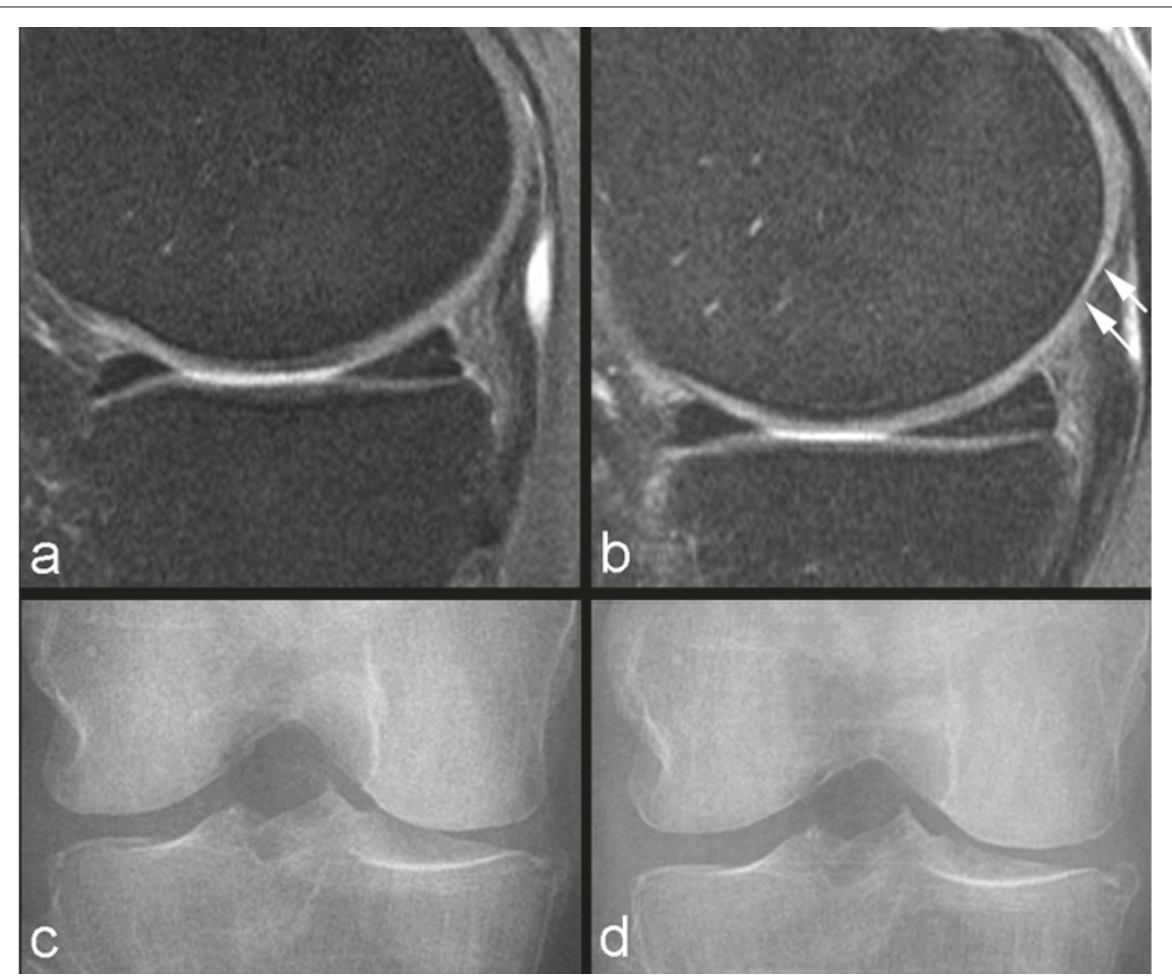

Figure 4. Example of non-sensitivity of radiography. (a) Baseline sagittal intermediate-weighted fat-suppressed image shows normal articular cartilage coverage in the medial femur and tibia. (b) At 24-month follow-up, there is circumscribed thinning of cartilage in the posterior medial femur (arrows). (c) Baseline radiograph does not show any abnormalities in regard to joint space width or any definite osteophytes at the medial tibia (no arrow). (d) At 24-month follow-up, no change is observed in comparison with the baseline image.

charge, mobile ions such as sodium $\left(\mathrm{Na}^{+}\right)$and charged gadolinium-based contrast agents for MRI (for example, gadolinium diethylene triamine pentaacetic acid (GdDTPA) ${ }^{2-}$ ) are distributed in relation to the proteoglycan concentration in cartilage, with $\mathrm{Na}^{+}$lower in concentration and Gd-DTPA ${ }^{2-}$ higher in concentration in glycosaminoglycan-depleted cartilage relative to normal tissue. Because collagen- and proteoglycan-associated glycosaminoglycans are important to the functional and structural integrity of cartilage, compositional MRI of cartilage is focused on collagen and glycosaminoglycan content [34]. MRI techniques for assessing the compositional characteristics of articular cartilage in the knee include T2 mapping, delayed gadolinium-enhanced magnetic resonance imaging of cartilage (dGEMRIC), T1 rho imaging, sodium imaging, and diffusion-weighted imaging (DWI).

T2 mapping is perhaps the most studied parameter for molecular imaging of cartilage. T2 is a reflection of the interactions between water molecules and surrounding macromolecules and is affected by many physiologic and pathophysiologic processes related to the state of cartilage. Focal increase in T2 relaxation time has been associated with cartilage matrix damage, particularly with loss of collagen integrity and an increase in water content [35-37]. T2 mapping techniques do not use contrast material, have been shown to be clinically useful, and are well validated and robust [3]. T2 maps may be used to monitor the effectiveness of cartilage repair over time [38]. T2 mapping can be implemented relatively easily since software for generating color T2 maps is now available in commercial packages [3].

$\mathrm{T} 1 \mathrm{rho}$, like $\mathrm{T} 2$, is sensitive to interactions of water with macromolecules. T1 rho has been shown to correlate with the proteoglycan concentration in cartilage [39]. A recent study demonstrated that changes of $\mathrm{T} 1$ rho with loading may be related to cartilage biomechanical properties (that is, elasticity) and may be a valuable tool for identifying early cartilage disease [40].

The delayed gadolinium-enhanced magnetic resonance imaging of cartilage (dGEMRIC) technique is based on the fact that glycosaminoglycans contain negatively charged side chains, which lead to an inverse distribution of negatively charged contrast agent molecules (such as gadolinium) with respect to glycosaminoglycans concentration. As the concentration of Gd-DTPA ${ }^{2-}$ molecules can be approximated by measuring T1, T1 mapping of cartilage after intravenous administration of Gd-DTPA ${ }^{2-}$ allows a quantitative assessment of glycosaminoglycan content $[3,34]$. The T1 measurement after penetration of 


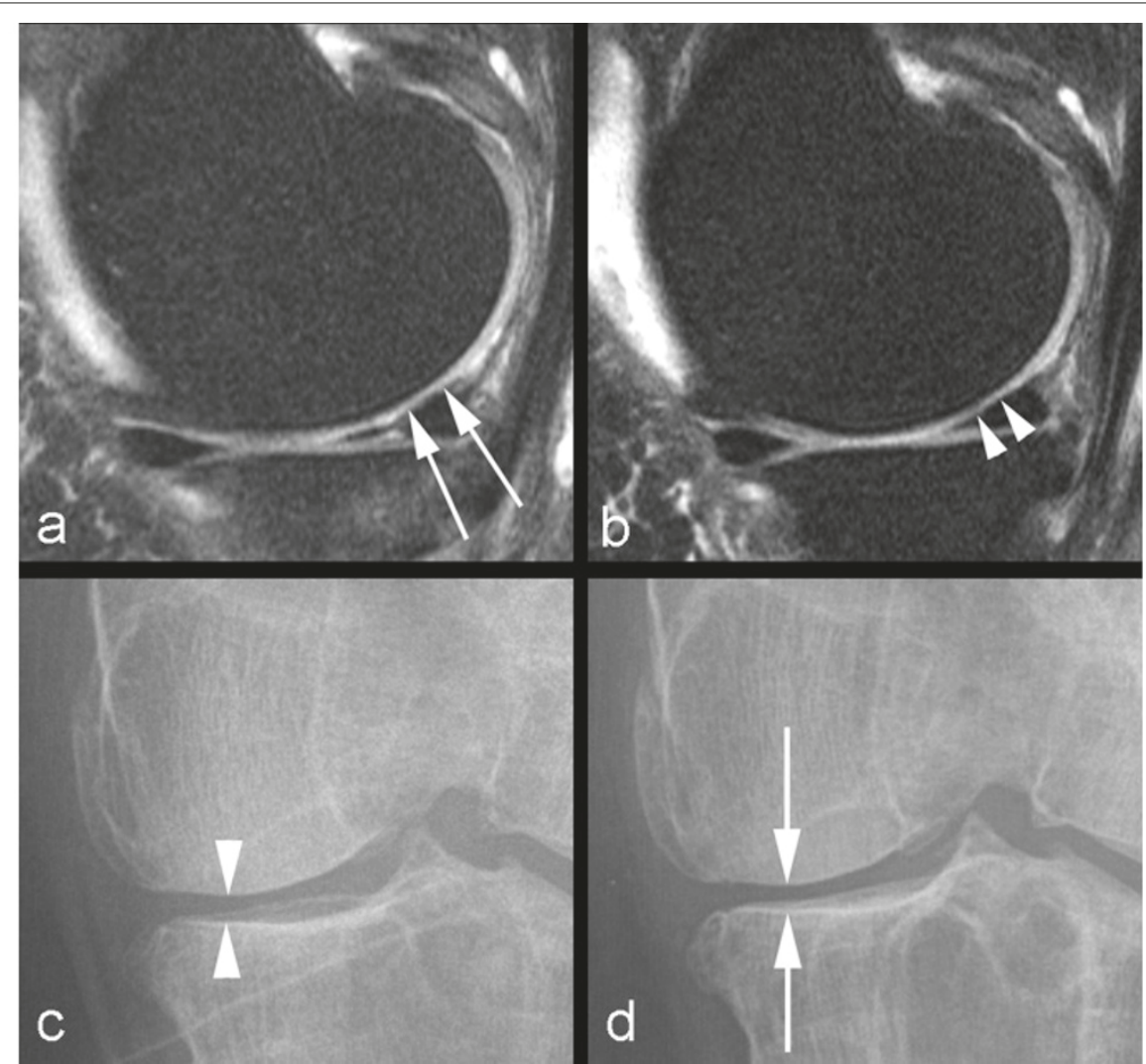

Figure 5. Example of non-specificity of radiography. (a) Baseline sagittal intermediate-weighted fat-suppressed image shows discrete superficial cartilage loss at the central part of the medial femur (arrows). (b) No progression is seen at 24-month follow-up (arrowheads). (c) Radiographic joint space narrowing is depicted at baseline (arrowheads). (d) Further decrease in joint space width is shown at 24-month follow-up (arrows) and was due to meniscal extrusion (not shown).

Gd-DTPA $^{2-}$ is referred to as the dGEMRIC index. Areas of cartilage with a lower dGEMRIC index are commonly observed in joints in which radiographic findings are indicative of OA [41]. The dGEMRIC index has been validated against histologic and biochemical measurements of glycosaminoglycan content in clinical studies [42]. dGEMRIC has potential as a non-invasive technique for monitoring the glycosaminoglycan content of cartilage after repair by various techniques [43] (Figure 6). It has also shown the ability to demonstrate changes in morphologically intact cartilage that may be predictive of progression to OA [44]. A recent pilot randomized controlled trial showed that the dGEMRIC technique may be able to detect changes in proteoglycan content in knee cartilage among individuals taking collagen hydrolysate after 24 weeks [45]. Drawbacks of the dGEMRIC technique are the double dose of MRI contrast agent that is needed and the delay between intravenous contrast administration and MRI examination to allow complete penetration of the contrast agent into the cartilage.

Sodium is a naturally abundant positively charged ion that will distribute itself in cartilage in proportion to the concentration of negatively charged glycosaminoglycan molecules. Sodium MRI has demonstrated promising results in the compositional assessment of articular cartilage. It can depict regions of proteoglycan depletion, which exhibit lower signal intensity than do areas of normal cartilage [46-48]. Thus, sodium MRI may be useful for differentiating between early-stage degenerated cartilage and normal cartilage [48]. Although sodium MRI has high specificity and does not require any exogenous contrast agent, it does require special hardware capabilities (multinuclear), specialized radiofrequency coils (transmit/receive), and likely 3D ultrashort TE sequences. These challenges currently limit the use of sodium MRI in a clinical setting.

Lastly, DWI of cartilage is based on the motion of water molecules within cartilage. The magnitude and direction of local tissue diffusivity are related to the macromolecular environment of bulk water diffusion. The diffusion of water in articular cartilage reflects the biochemical structure and architecture of the tissue. The apparent diffusion coefficient (ADC) is low at long diffusion times in healthy cartilage because the diffusion of water molecules is restricted by cartilage components. 


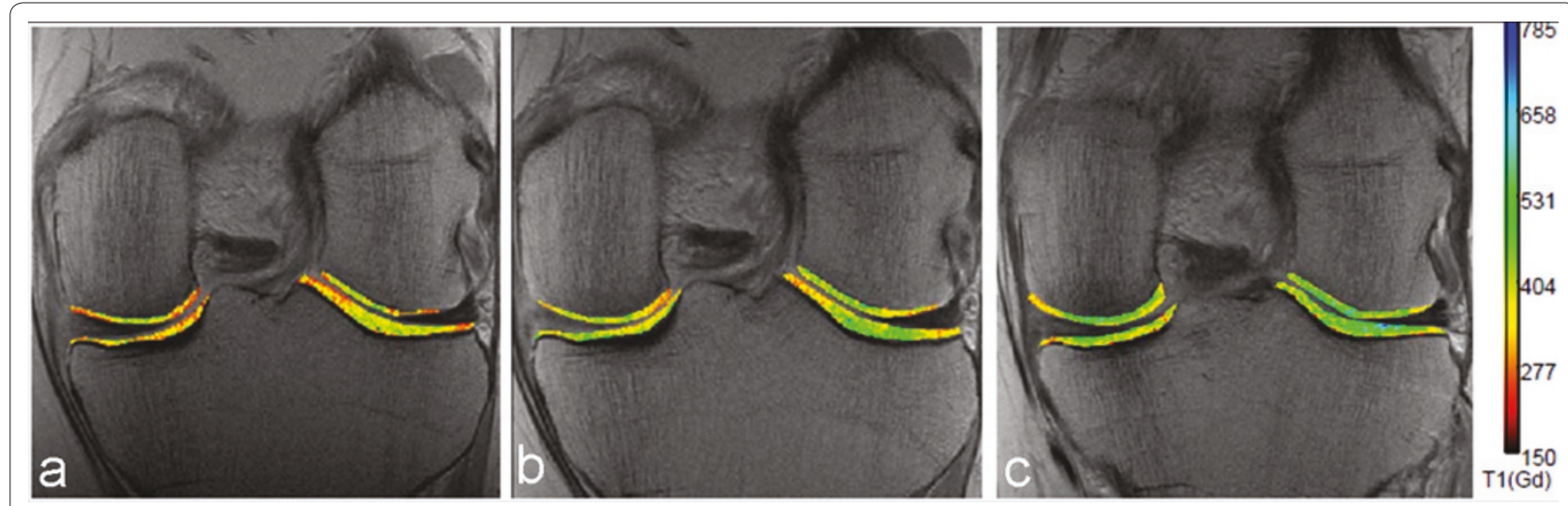

Figure 6. dGEMRIC images of knees in an individual without evidence of radiographic osteoarthritis (Kellgren-Lawrence grade 0). (a) Before, (b) 3 months after, and (c) 1 year after meniscal repair surgery. The color scale shows values increasing from lower values (red-yellow range) to mid-high values (yellow-green range) over this time period. These images demonstrate the potential to show variations in cartilage molecular status even in regions of morphologically intact tissue and, as in this case, potentially monitor cartilage repair when relieved of trauma or with an intervention designed to improve the biomechanical status of the joint. dGEMRIC, delayed gadolinium-enhanced magnetic resonance imaging of cartilage.

However, disruption of the cartilage matrix results in enhanced water mobility, which increases the ADC values of cartilage [49]. A recent study of DWI in the assessment of cartilage repair such as autologous chondrocyte implantation in vivo reported good sensitivity for tracking changes in the diffusion behavior of implants over time [50]. The last two techniques (that is, sodium imaging and DWI) are still in their infancy; to our knowledge, no large clinical studies using either technique in patients with knee OA or knee cartilage repair have been published. However, the field of compositional MRI seems to have great potential for increasing our understanding of OA and for the development of diseasemodifying treatments.

\section{Performance of magnetic resonance imaging}

A recent systematic review by Hunter and colleagues [51] revealed that MRI as an outcome measure in OA provided strong support for both its concurrent and predictive validity. MRI cartilage findings were associated with CT arthrographic, histologic, and arthroscopic findings, but the relation of cartilage volume and thickness and compositional measures to the presence of radiographic OA was inconsistent [51]. The literature review also demonstrated that cartilage pathology can be measured reliably and with good responsiveness by using a semiquantitative or quantitative technique [26]. Quantitative cartilage volume change and presence of cartilage defects are potential predictors of total knee replacement [51].

\section{Limitations of magnetic resonance imaging}

It is critically important to acquire MRI by using appropriate pulse sequences tailored to the specific purpose of cartilage assessment. For example, for evaluation of focal cartilage defects, fluid-sensitive sequences such as fat suppression intermediate-weighted, proton densityweighted, or T2-weighted FSE sequences should be used [52-54]. In contrast, GRE sequences such as 3D spoiled gradient echo at a steady state (SPGR), fast low angle shot (FLASH), 3-point Dixon, and double echo steady state (DESS) are well suited for quantitative (volumetric) analysis of cartilage $[55,56]$. Use of inappropriate pulse sequences will inevitably invalidate the results of a study [57]. Expert musculoskeletal radiologists who can ensure the use of adequate imaging protocol may be available in only a limited number of institutions. MRI assessment, whether a simple visual inspection or a semiquantitative or quantitative assessment, is a time-consuming task and needs to be performed by trained readers. Centralized reading of MRI by expert readers seems to be essential to maintain standardized assessment. Some of the techniques may be vendor-specific, and sophisticated MRI analyses (such as volumetric segmentations, dGEMRIC, and sodium MRI) require specialized software and can be done only at selected institutions.

Additional limitations of MRI include (a) difficulties with repositioning in MRI (for example, in the evaluation of knee structures by semiquantitative scoring and when using dGEMRIC in two dimensions longitudinally), (b) challenges of having obese patients who might be too obese to fit the coil (resulting in decreased signal-to-noise ratio), and (c) the fact that different hospitals are equipped with MRI systems from various vendors and the use of the identical pulse sequences across all sites may not be possible in multicenter MRI studies. 
Table 1. Strengths and limitations of radiography and magnetic resonance imaging

\begin{tabular}{|c|c|c|}
\hline & Radiography & Magnetic resonance imaging \\
\hline \multirow[t]{4}{*}{ Strengths } & - Inexpensive & - $\quad$ Sensitive to change \\
\hline & - Widely available & - $\quad$ Specific because it directly shows cartilage \\
\hline & - $\quad$ Short examination time & $\begin{array}{l}\text { - Positioning is not an issue, since cartilage can be directly } \\
\text { visualized }\end{array}$ \\
\hline & & - Treats the joint as a whole organ \\
\hline \multirow[t]{7}{*}{ Limitations } & $\begin{array}{l}\text { - } \quad \text { Not sensitive to change: minimal progression is not } \\
\text { identifiable, so large patient sample size and lengthy studies } \\
\text { are required }\end{array}$ & $\begin{array}{l}\text { - } \quad \text { Expensive } \\
\text { - } \quad \text { Limited availability }\end{array}$ \\
\hline & \multirow{2}{*}{$\begin{array}{l}\text { - Not specific: meniscal extrusion and damage may account } \\
\text { for joint space narrowing }\end{array}$} & - $\quad$ Long examination time \\
\hline & & - $\quad$ Requires expert knowledge (that is, input of radiologist is \\
\hline & $\begin{array}{l}\text { Change in positioning is problematic in multicenter and } \\
\text { long-term studies }\end{array}$ & $\begin{array}{l}\text { essential) for choice of appropriate pulse sequences and imaging } \\
\text { protocol }\end{array}$ \\
\hline & \multirow{3}{*}{$\begin{array}{l}\text { Does not show other important structures that account for } \\
\text { pain in osteoarthritis }\end{array}$} & - $\quad$ Difficulties with repositioning \\
\hline & & - $\quad$ Challenges of imaging obese patients who do not fit the coil \\
\hline & & $\begin{array}{l}\text { Images with identical pulse sequences may not be obtained } \\
\text { across all sites in multicenter magnetic resonance imaging } \\
\text { studies }\end{array}$ \\
\hline
\end{tabular}

\section{Conclusions}

Radiography is severely limited by its inability to directly visualize cartilage, the difficulty of ascertaining the optimum and reproducible positioning of the joint in serial assessments, and the difficulty of grading JSN by visual evaluation. MRI enables visualization of not only cartilage but also other important features of OA simultaneously. Strengths and limitations of radiography and MRI are summarized in Table 1. 'Pre-radiographic' cartilage changes depicted by MRI can be measured reliably by using a semiquantitative or quantitative approach. MRI enables accurate measurement of longitudinal change in quantitative cartilage morphology in knee OA. Moreover, compositional MRI allows imaging of 'premorphologic' changes (that is, visualization of subtle intrasubstance matrix changes before any obvious morphologic alterations occur). Our research focus has shifted from detection of JSN on radiography to visualization of morphologic and pre-morphologic changes of cartilage by using conventional as well as sophisticated MRI techniques. To take full advantage of these new research tools, consensus-driven MRI-based definitions of OA diagnosis and grading of severity need to be established and validated, and inclusion of MRI in a new regulatory guidance statement for DMOAD clinical trials should follow promptly.

This article is part of the series Advances in the imaging of rheumatic diseases, edited by Mikkel Østergaard. Other articles in this series can be found at http://arthritis-research.com/series/imaging

\section{Abbreviations}

3D, three-dimensional; $A D C$, apparent diffusion coefficient; $A P$, anteroposterior; dGEMRIC, delayed gadolinium-enhanced magnetic resonance imaging of cartilage; DMOAD, disease-modifying osteoarthritis drug; DWI, diffusion-weighted imaging; FDA, US Food and Drug Administration; FSE, fast spin echo; Gd-DTPA, gadolinium diethylene triamine pentaacetic acid; GRE, gradient-recalled echo; ISN, joint space narrowing; ISW joint space width; KL, Kellgren-Lawrence; MMP, matrix metalloproteinase; MRI, magnetic resonance imaging; OA, osteoarthritis; OAl, Osteoarthritis Initiative; OARSI, Osteoarthritis Research Society International; SE, spin echo; WORMS, Whole Organ Magnetic Resonance Imaging Score.

\section{Competing interests}

AG is the president of Boston Imaging Core Lab, LLC (Boston, MA, USA) and a consultant to Merck Serono (Geneva, Switzerland), Facet Solutions (Hopkinton, MA, USA), Novartis (Basel, Switzerland), Genzyme Corporation (Cambridge, MA, USA), and Stryker (Kalamazoo, MI, USA) and received research support from GE Healthcare (Little Chalfont, Buckinghamshire, UK). FWR holds shares in Boston Imaging Core Lab, LLC. The other authors declare that they have no competing interests.

\section{Authors' contributions}

All authors participated in the conception and design, analysis and interpretation of the data, drafting of the article, and critical revision of the article for important intellectual content. All authors read and approved the final manuscript.

\section{Author details}

'Quantitative Imaging Center, Department of Radiology, Boston University School of Medicine, 820 Harrison Avenue, FGH Building, 3rd Floor, Boston, MA 02118, USA. ²Department of Radiology, Beth Israel Deaconess Medical Center, Harvard Medical School, 330 Brookline Avenue, Boston, MA 02215 , USA.

Published: 24 November 2011

\section{References}

1. Hunter DJ, Zhang YQ, Tu X, Lavalley M, Niu JB, Amin S, Guermazi A, Genant H, Gale D, Felson DT: Change in joint space width: hyaline articular cartilage loss or alteration in meniscus? Arthritis Rheum 2006, 54:2488-2495.

2. Hellio Le Graverand M-P, Mazzuca S, Duryea J, Brett A: Radiographic-based grading methods and radiographic measurement of joint space width in 
osteoarthritis. Radiol Clin N Am 2009, 47:567-579.

3. Crema MD, Roemer FW, Marra MD, Burstein D, Gold GE, Eckstein F, Baum T, Mosher TJ, Carrino JA, Guermazi A: Articular cartilage in the knee: current MR imaging techniques and applications in clinical practice and research. Radiographics 2011, 31:37-61.

4. Kellgren JH, Lawrence JS: Radiological assessment of osteoarthritis. Ann Rheum Dis 1957, 16:494-502.

5. Guermazi A, Hunter DJ, Roemer FW: Plain radiography and magnetic resonance imaging diagnostics in osteoarthritis: validated staging and scoring. Bone Joint Surg Am 2009, 91:54-62.

6. Altman RD, Gold GE: Atlas of individual radiographic features in osteoarthritis, revised. Osteoarthritis Cartilage 2007, 15 Suppl A:A1-56.

7. Duryea J, Neumann G, Niu J, Totterman S, Tamez J, Dabrowski C, Le Graverand MP, Luchi M, Beals CR, Hunter DJ: Comparison of radiographic joint space width with magnetic resonance imaging cartilage morphometry: analysis of longitudinal data from the Osteoarthritis Initiative. Arthritis Care Res (Hoboken) 2010, 62:932-937.

8. Conaghan PG, Hunter DJ, Maillefert JF, Reichmann WM, Losina E: Summary and recommendations of the OARSI FDA osteoarthritis Assessment of Structural Change Working Group. Osteoarthritis Cartilage 2011, 19:606-610.

9. Mazzuca SA, Brandt KD, Lane KA, Katz BP: Knee pain reduces joint space width in conventional standing anteroposterior radiographs of osteoarthritic knees. Arthritis Rheum 2002, 46:1223-1227.

10. Kothari M, Guermazi A, von Ingersleben G, Miaux Y, Sieffert M, Block JE, Stevens R, Peterfy CG: Fixed-flexion radiography of the knee provides reproducible joint space width measurements in osteoarthritis. Eur Radiol 2004, 14:1568-1573.

11. Mazzuca SA, Brandt KD, Buckland-Wright JC, Buckwalter KA, Katz BP, Lynch JA, Ward RJ, Emsley CL: Field test of the reproducibility of automated measurements of medial tibiofemoral joint space width derived from standardized knee radiographs. J Rheumatol 1999, 26:1359-1365.

12. Buckland-Wright JC, Macfarlane DG, Williams SA, Ward RJ: Accuracy and precision of joint space width measurements in standard and macroradiographs of osteoarthritic knees. Ann Rheum Dis 1995, 54:872-880.

13. Guermazi A, Hunter DJ, Li L, Benichou O, Eckstein F, Kwoh CK, Nevitt M, Hayashi D: Different thresholds for detecting osteophytes and joint space narrowing exist between the site investigators and the centralized reader in a multicenter knee osteoarthritis study-data from the Osteoarthritis Initiative. Skeletal Radiol 2011 Apr 9. [Epub ahead of print].

14. Amin S, LaValley MP, Guermazi A, Grigoryan M, Hunter DJ, Clancy M, Niu J, Gale DR, Felson DT: The relationship between cartilage loss on magnetic resonance imaging and radiographic progression in men and women with knee osteoarthritis. Arthritis Rheum 2005, 52:3152-3159.

15. Gale DR, Chaisson CE, Totterman SM, Schwartz RK, Gale ME, Felson D: Meniscal subluxation: association with osteoarthritis and joint space narrowing. Osteoarthritis Cartilage 1999, 7:526-532.

16. Adams JG, MCAlindon T, Dimasi M, Carey J, Eustace S: Contribution of meniscal extrusion and cartilage loss to joint space narrowing in osteoarthritis. Clin Radiol 1999, 54:502-506.

17. Sharma L: Comment on: Varus malalignment negates the structuremodifying benefits of doxycycline in obese women with knee osteoarthritis. Osteoarthritis Cartilage 2010, 18:1006-1007.

18. Hayashi D, Guermazi A, Crema MD, Roemer FW: Imaging in osteoarthritis: what have we learned and where are we going? Minerva Med 2011, 102:15-32.

19. Hayashi D, Guermazi A, Hunter DJ: Osteoarthritis year 2010 in review: imaging. Osteoarthritis Cartilage 2011, 19:354-360.

20. Peterfy CG, Guermazi A, Zaim S, Tirman PF, Miaux Y, White D, Kothari M, LuY, Fye K, Zhao S, Genant HK: Whole-Organ Magnetic Resonance Imaging Score (WORMS) of the knee in osteoarthritis. Osteoarthritis Cartilage 2004, 12:177-190

21. Hunter DJ, Lo GH, Gale D, Grainger AJ, Guermazi A, Conaghan PG: The reliability of a new scoring system for knee osteoarthritis MRI and the validity of bone marrow lesion assessment: BLOKS (Boston Leeds Osteoarthritis Knee Score). Ann Rheum Dis 2008, 67:206-211.

22. Kornaat PR, Ceulemans RY, Kroon HM, Riyazi N, Kloppenburg M, Carter WO, Woodworth TG, Bloem JL: MRI assessment of knee osteoarthritis: Knee Osteoarthritis Scoring System (KOSS) - inter-observer and intra-observer reproducibility of a compartment-based scoring system. Skeletal Radiol 2005, 34:95-102.

23. Roemer FW, Guermazi A: MR imaging-based semiquantitative assessment in osteoarthritis. Radiol Clin North Am 2009, 47:633-654.

24. Hunter DJ, Guermazi A, Lo GH, Grainger AJ, Conaghan PG, Boudreau RM, Roemer FW: Evolution of semi-quantitative whole joint assessment of knee OA: MOAKS (MRI Osteoarthritis Knee Score). Osteoarthritis Cartilage 2011, 19:990-1002

25. Roemer FW, Zhang Y, Niu J, Lynch JA, Crema MD, Marra MD, Nevitt MC, Felson DT, Hughes LB, El-Khoury GY, Englund M, Guermazi A; Multicenter Osteoarthritis Study Investigators: Tibiofemoral joint osteoarthritis: risk factors for MR-depicted fast cartilage loss over a 30-month period in the multicenter osteoarthritis study. Radiology 2009, 252:772-780.

26. Hunter DJ, Zhang W, Conaghan PG, Hirko K, Menashe L, Reichmann WM Losina E: Responsiveness and reliability of MRI in knee osteoarthritis: a meta-analysis of published evidence. Osteoarthritis Cartilage 2011, 19:589-605.

27. Buck RJ, Wyman BT, Le Graverand MP, Wirth W, Eckstein F: An efficient subset of morphological measures for articular cartilage in the healthy and diseased human knee. Magn Reson Med 2010, 63:680-690.

28. Buck RJ, Wyman BT, Le Graverand MP, Hudelmaier M, Wirth W, Eckstein F: Does the use of ordered values of subregional change in cartilage thickness improve the detection of disease progression in longitudinal studies of osteoarthritis? Arthritis Rheum 2009, 61:917-924.

29. Wirth W, Buck R, Nevitt M, Le Graverand MP, Benichou O, Dreher D, Davies RY, Lee JH, Picha K, Gimona A, Maschek S, Hudelmaier M, Eckstein F; OAl Investigators: MRI-based extended ordered values more efficiently differentiate cartilage loss in knees with and without joint space narrowing than region-specific approaches using MRI or radiography data from the OA initiative. Osteoarthritis Cartilage 2011, 19:689-699.

30. Ding C, Cicuttini F, Jones G: Do NSAIDs affect longitudinal changes in knee cartilage volume and knee cartilage defects in older adults? Am J Med 2009, 122:836-842

31. Berry PA, Maciewicz RA, Cicuttini FM, Jones MD, Hellawell CJ, Wluka AE: Markers of bone formation and resorption identify subgroups of patients with clinical knee osteoarthritis who have reduced rates of cartilage loss. J Rheumatol 2010, 37:1252-1259.

32. Berry PA, Maciewicz RA, Wluka AE, Downey-Jones MD, Forbes A, Hellawell CJ, Cicuttini FM: Relationship of serum markers of cartilage metabolism to imaging and clinical outcome measures of knee joint structure. Ann Rheum Dis 2010, 69:1816-1822.

33. Wildi LM, Raynauld JP, Martel-Pelletier J, Abram F, Dorais M, Pelletier JP: Relationship between bone marrow lesions, cartilage loss and pain in knee osteoarthritis: results from a randomised controlled clinical trial using MRI. Ann Rheum Dis 2010, 69:2118-2124.

34. Burstein D, Gray M, Mosher T, Dardzinski B: Measures of molecular composition and structure in osteoarthritis. Radiol Clin North Am 2009, 47:675-686.

35. Menezes NM, Gray ML, Hartke JR, Burstein D: T2 and T1 rho MRI in articular cartilage systems. Magn Reson Med 2004, 51:503-509.

36. Dunn TC, Lu Y, Jin H, Ries MD, Majumdar S: T2 relaxation time of cartilage at MR imaging: comparison with severity of knee osteoarthritis. Radiology 2004, 232:592-598

37. Jones EF, Schooler J, Miller DC, Drake CR, Wahnishe H, Siddiqui S, Li X, Majumdar S: Characterization of Human Osteoarthritic Cartilage Using Optical and Magnetic Resonance Imaging. Mol Imaging Biol 2011 Mar 8. [Epub ahead of print].

38. Welsch GH, Mamisch TC, Domayer SE, Dorotka R, Kutscha-Lissberg F, Marlovits S, White LM, Trattnig S: Cartilage T2 assessment at 3-T MR imaging: in vivo differentiation of normal hyaline cartilage from reparative tissue after two cartilage repair procedures - initial experience. Radiology 2008, 247:154-161

39. Borthakur A, Mellon E, Niyogi S, Witschey W, Kneeland JB, Reddy R: Sodium and T1 rho MRI for molecular and diagnostic imaging of articular cartilage. NMR Biomed 2006, 19:781-821.

40. Souza RB, Stehling C, Wyman BT, Hellio Le Graverand MP, Li X, LinkTM, Majumdar S: The effects of acute loading on T1rho and T2 relaxation times of tibiofemoral articular cartilage. Osteoarthritis Cartilage 2010, 18:1557-1563.

41. Williams A, Sharma L, McKenzie CA, Prasad PV, Burstein D: Delayed gadolinium-enhanced magnetic resonance imaging of cartilage in knee osteoarthritis: findings at different radiographic stages of disease and relationship to malalignment. Arthritis Rheum 2005, 52:3528-3535.

42. Bashir A, Gray ML, Hartke J, Burstein D: Nondestructive imaging of human 
cartilage glycosaminoglycan concentration by MRI. Magn Reson Med 1999, 41:857-865.

43. Watanabe A, Wada Y, Obata T, Ueda T, Tamura M, Ikehira H, Moriya H: Delayed gadolinium-enhanced MR to determine glycosaminoglycan concentration in reparative cartilage after autologous chondrocyte implantation: preliminary results. Radiology 2006, 239:201-208.

44. Owman H, Tiderius CJ, Neuman P, Nyquist F, Dahlberg LE: Association between findings on delayed gadolinium-enhanced magnetic resonance imaging of cartilage and future knee osteoarthritis. Arthritis Rheum 2008, 58:1727-1730.

45. McAlindon TE, Nuite M, Krishnan N, Ruthazer R, Price LL, Burstein D, Griffith J, Flechsenhar K: Change in knee osteoarthritis cartilage detected by delayed gadolinium enhanced magnetic resonance imaging following treatment with collagen hydrolysate: a pilot randomized controlled trial. Osteoarthritis Cartilage 2011, 19:399-405.

46. Wang L, Wu Y, Chang G, Oesingmann N, Schweitzer ME, Jerschow A, Regatte RR: Rapid isotropic 3D-sodium MRI of the knee joint in vivo at 7T. J Magn Reson Imaging 2009, 30:606-614.

47. Wheaton AJ, Borthakur A, Shapiro EM, Regatte RR, Akella SV, Kneeland JB, Reddy R: Proteoglycan loss in human knee cartilage: quantitation with sodium MR imaging - feasibility study. Radiology 2004, 231:900-905.

48. Borthakur A, Shapiro EM, Beers J, Kudchodkar S, Kneeland JB, Reddy R: Sensitivity of MRI to proteoglycan depletion in cartilage: comparison of sodium and proton MRI. Osteoarthritis Cartilage 2000, 8:288-293.

49. Burstein D, Gray ML, Hartman AL, Gipe R, Foy BD: Diffusion of small solutes in cartilage as measured by nuclear magnetic resonance (NMR) spectroscopy and imaging. J Orthop Res 1993, 11:465-478.

50. Mamisch TC, Menzel MI, Welsch GH, Bittersohl B, Salomonowitz E, Szomolanyi P, Kordelle J, Marlovits S, Trattnig S: Steady-state diffusion imaging for MR in-vivo evaluation of reparative cartilage after matrix-associated autologous chondrocyte transplantation at 3 tesla - preliminary results. Eur J Radiol 2008, 65:72-79.
51. Hunter DJ, Zhang W, Conaghan PG, Hirko K, Menashe L, Li L, Reichmann WM Losina E: Systematic review of the concurrent and predictive validity of MRI biomarkers in OA. Osteoarthritis Cartilage 2011, 19:557-588.

52. Barr C, Bauer JS, Malfair D, Ma B, Henning TD, Steinbach L, Link TM: MR imaging of the ankle at 3 Tesla and 1.5 Tesla: protocol optimization and application to cartilage, ligament and tendon pathology in cadaver specimens. Eur Radiol 2007, 17:1518-1528.

53. Bauer JS, Barr C, Henning TD, Malfair D, Ma CB, Steinbach L, LinkTM: Magnetic resonance imaging of the ankle at 3.0 Tesla and 1.5 Tesla in human cadaver specimens with artificially created lesions of cartilage and ligaments. Invest Radio/ 2008, 43:604-611.

54. Roemer FW, Kwoh CK, Hannon MJ, Crema MD, Moore CE, Jakicic JM, Green SM, Guermazi A: Semiquantitative assessment of focal cartilage damage at 3T MRI: A comparative study of dual echo at steady state (DESS) and intermediate-weighted (IW) fat suppressed fast spin echo sequences. Eur J Radiol 2011, 80:e126-131.

55. Bauer JS, Krause SJ, Ross CJ, Krug R, Carballido-Gamio J, Ozhinsky E, Majumdar S, Link TM: Volumetric cartilage measurements of porcine knee at 1.5-T and 3.0-T MR imaging: evaluation of precision and accuracy. Radiology 2006, 241:399-406.

56. Eckstein F, Burstein D, LinkTM: Quantitative MRI of cartilage and bone: degenerative changes in osteoarthritis. NMR Biomed 2006, 19:822-854

57. Hayashi D, Roemer FW, Guermazi A: Choice of pulse sequences for magnetic resonance imaging-based semiquantitative assessment of cartilage defects in osteoarthritis research: comment on the article by Dore et al. Arthritis Rheum 2010, 62:3830-3831; author reply 3831-3832.

doi:10.1186/ar3488

Cite this article as: Guermazi $A$, et al: Why radiography should no longer be considered a surrogate outcome measure for longitudinal assessment of cartilage in knee osteoarthritis. Arthritis Research \& Therapy 2011, 13:247. 